Chirurg 2015 $\cdot 86: 183$

DOI 10.1007/s00104-015-2987-z

Online publiziert: 28. Januar 2015

๑) Springer-Verlag Berlin Heidelberg 2015

O. Strobel · M.W. Büchler

Klinik für Allgemein-, Viszeral- und Transplantationschirurgie, Universität Heidelberg

\title{
Nach neoadjuvanter Therapie eines Pankreaskarzinoms sind die klassischen Resektabilitätskriterien aus der Schnittbildgebung nicht mehr anwendbar
}

vorlagen (bis kurzstreckiger Verschluss der Pfortader/VMS, Ummauerung der A. gastroduodenalis bis zur A. hepatica, $<180^{\circ}$-Kontakt zur AMS). Die Bildgebung vor und nach nTx wurde durch einen erfahrenen, bezüglich der Therapie verblindeten Pankreaschirurgen beurteilt.

\section{Ergebnisse}

Von 47 Patienten mit nTx mit FOLFIRINOX hatten 24 (51\%) zusätzlich eine Radiochemotherapie erhalten. Patienten mit nTx waren jünger, hatten einen besseren Performance-Status und einen niedrigeren Body Mass Index als Patienten mit primärer Resektion. Unter nTx wurde als Zeichen eines Ansprechens ein Abfall des CA (Carbohydrate-Antigen) 199 und eine Tumorverkleinerung in der Bildgebung (von 3,6 auf 2,2 cm) beobachtet. Trotzdem persistierten bei 19 (von initial 26) Patienten die radiologischen Kriterien der Irresektabilität und bei 9 (von initial 14) Patienten die Kriterien der Borderline-Resektabilität. Nur 12 Patienten hatten nach nTx anhand der Bildgebung resektable Tumoren. Trotzdem konnte bei 40 Patienten eine Resektion durchgeführt werden, davon 35 R0-Resektionen. $7 \mathrm{~Pa}$ tienten wurden bei Metastasen $(n=3)$ oder persistierender Irresektabilität $(n=4)$ nur exploriert. Bei 2 Patienten lag eine komplette pathologische Response vor. Positive Lymphknoten waren nach nTx signifikant seltener (35\% vs. $79 \%$ ) und die Morbidität deutlich niedriger als bei primärer Resektion (36\% vs. 63\%).

\section{Diskussion}

Die Autoren folgern, dass nach nTx mit FOLFIRINOX die für die primäre Resek- tion geltenden Resektabilitätskriterien aus der Schnittbildgebung nicht mehr anwendbar sind.

Dies könnte auch für andere neoadjuvante Therapieregime gelten. Insbesondere darf das Potenzial der neoadjuvanten Radiochemotherapie (hier bei $51 \%$ zusätzlich durchgeführt) nicht unterschätzt werden. Vermutlich gelten die radiologischen Resektabilitätskriterien nicht mehr, weil die Schnittbildgebung nicht zwischen stromareichem Tumorgewebe und bindegewebiger Narbe unterscheiden kann.

Bei Patienten mit primär irresektablen Tumoren, bei denen es unter neoadjuvanter (oder palliativer) Therapie zu einer biologischen Response (CA19-9-Abfall) kommt und bei denen kein Anhalt für Metastasen besteht, sollte daher auch bei unverändertem Lokalbefund die chirurgische Exploration mit dem Ziel der Resektion erwogen werden.

Zur Wertigkeit einer nTx mit FOLFIRINOX vs. primärer Resektion bei (Borderline-)resektablen Tumoren erlaubt die vorliegende Arbeit aufgrund ihres Designs keinerlei Schlüsse. Dies kann nur in randomisiert kontrollierten Studien geklärt werden.

\section{Korrespondenzadresse}

PD Dr. O. Strobel

Klinik für Allgemein-, Viszeralund Transplantationschirurgie, Universität Heidelberg,

Im Neuenheimer Feld 110, 69120 Heidelberg Oliver.Strobel@med.uni-heidelberg.de

Interessenkonflikt. O. Strobel und M.W. Büchler geben an, dass kein Interessenkonflikt besteht.
[AMS], Truncus coeliacus oder A hepatica) oder der Borderline-Resektabilität 